> Les firmes Zeiss et Leitz sont nées toutes deux au milieu du XIX $x^{\mathrm{e}}$ siècle. Mais leurs trajectoires diffèrent radicalement. Tandis que Zeiss - comme nous l'avons vu précédemment [1] - est rapidement devenue une compagnie dirigée par les membres du conseil d'administration, la maison Leitz est longtemps restée une affaire familiale. Elle fut à l'origine du plus célèbre appareil photo du monde, le Leica, qui séduisit les plus grands reporters et devint «l'œil du $x x^{e}$ siècle ». Elle fut aussi, pendant la période nazie, dirigée par un « Juste », Ernst Leitz II, qui réussit à sauver plusieurs dizaines de Juifs allemands. Munis d'un billet pour New York et d'un visa de l'entreprise, ils échappaient ainsi à l'extermination. Ce n'est que bien plus tard que l'histoire fut divulguée sous le titre The Leica freedom train par Franck Dabba Smith, qui avait assisté à l'arrivée de ces garçons un peu perdus au magasin new yorkais sur la Ve avenue. <

\section{Grandeur \\ et vicissitudes \\ de deux grandes \\ compagnies \\ d'optique \\ allemandes}

\section{La firme Leitz}

Simone Gilgenkrantz

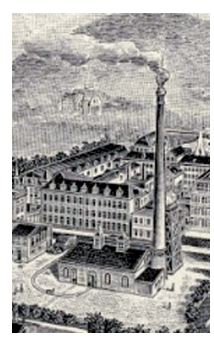

S. Gilgenkrantz :

9 , rue Basse,

54330 Clérey-sur-Brénon, France.

simsimone.gilgenkrantz@gmail.com

portantes améliorations à la fabrication des microscopes et des télescopes et, grâce à la qualité de ses oculaires, il se fait rapidement connaître des scientifiques de l'époque comme Carl Friedrich Gauss (1777-1855), réputé pour ses études biométriques, ou Justus von Liebig, chimiste et agronome (dont le nom reste encore aujourd'hui attaché à un potage). Bientôt, il doit embaucher du personnel pour fabriquer les centaines de microscopes qu'on lui commande. Quand il meurt de tuberculose à 28 ans en 1855, il a déjà douze employés.

Scandale dans cette petite ville conservatrice, sa veuve se remarie assez rapidement avec son associé Friedrich Belthe. Celui-ci cherche un ingénieur pour conserver à l'entreprise sa créativité. II s'adresse à Juncker, qui a travaillé pendant plusieurs années chez Mathaus Hypp², qui lui recommande un jeune ingénieur de la maison Hypp : Ernst Leitz. Ce dernier est embauché, devient associé à l'affaire, et la reprend à son compte au décès de Belthe en 1869.

\title{
L'Optisches Institut
}

C'est en 1848 que Carl Keller (1826-1855) monte une fabrique d'instruments d'optique dans la petite ville de Wetz$\operatorname{lar}^{1}$, au bord du Lahn, aujourd'hui dans le Land de Hesse. Il est le fils d'un directeur d'une usine de hauts-fourneaux. Après ses études, il fait un stage de mécanique à Giessen, puis travaille à Hambourg chez Repsold et fils, des pionniers dans la construction d'appareils scientifiques. Féru de technique et de mathématiques, il apporte d'im-

${ }^{1}$ C'est à partir de faits réels qui se sont déroulés à Wetzlar vers 1772 que Goethe conçut son roman «Les souffrances du jeune Werther ».

\section{Optisches Institut von Ernst Leitz}

Dans les années 1870, la cytologie et la bactériologie sont en plein essor et les demandes de microscopes sont nombreuses. Au début de l'ère industrielle, dans cette confédération de l'Allemagne du Nord qu'il vient de créer, Bismarck tend à mener une politique sociale afin d'apaiser certaines revendications.

${ }^{2}$ Ce génial horloger-électricien, qui a beaucoup travaillé en Suisse, a réalisé, entre autres, des horloges électriques, des chronomètres et des réseaux télégraphiques. 


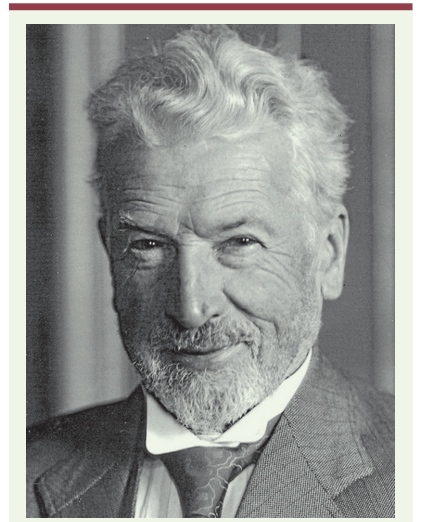

Portrait du fondateur, Ernst Leitz I

Ernst Leitz (1843-1920), protestant progressiste, met en œuvre pour ses employés cette politique sociale : possibilité d'acquérir une maison individuelle, une assurance maladie. II offre de hauts salaires et la sélection à l'embauche se fait sur la compétence. En se développant, la maison Leitz perfectionne ses microscopes dont les ventes continuent d'augmenter.

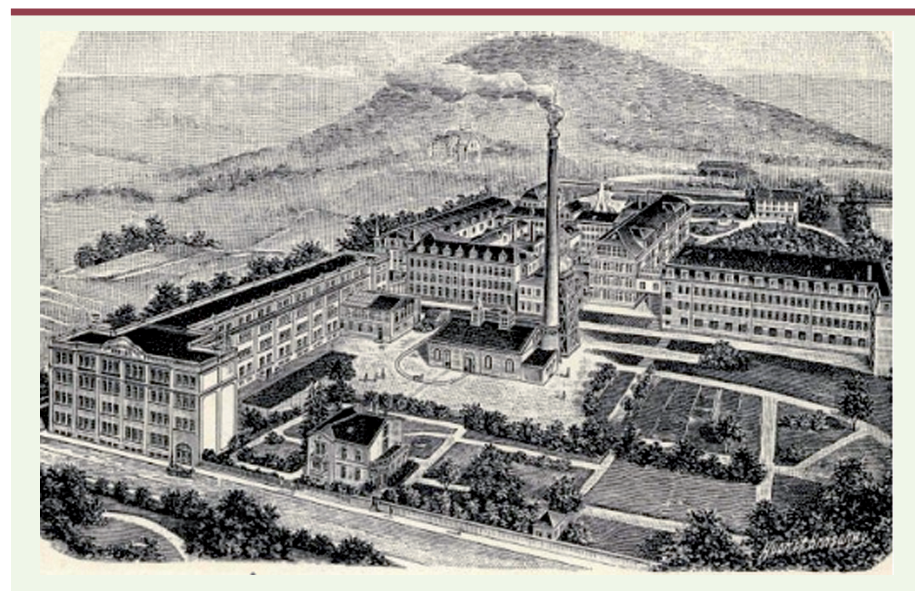

Les usines Leitz à Wetzlar vers 1910

Après de bonnes études, son fils Ernst Leitz junior (1871-1956) entre à l'usine et devient associé à l'âge de 35 ans. II poursuit la même politique sociale que son père (il connaît le prénom de tous les employés). Vers 1910, Ernst II souhaite diversifier encore la production et se lancer dans le cinématographe. Or, chez Zeiss, à léna, Emil Mechau travaille sur les projecteurs et fait des recherches pour supprimer l'effet de scintillement très gênant du temps du cinéma muet. Mais ses travaux ne sont guère encouragés. Déçu, il choisit donc de venir travailler chez Leitz qui a la réputation de laisser assez de liberté à ses ingénieurs. C'est sur ses conseils qu'Ernst II, qui souhaite aussi un chef de recherches en cinéma, embauche Oskar Barnack (1879-1936).

\section{La naissance du Leica}

Les films tournés au début $d u x x^{e}$ siècle ne donnent pas de résultats réguliers (en fonction des pellicules et de l'exposition à la lumière).
Un posemètre est utilisé afin de les étalonner. Oskar Barnack est confronté à ce problème et a l'idée de construire un boîtier pour déterminer le temps de pose et de développement des films. Il aime aussi faire de la photo en extérieur. Mais, à l'époque, les appareils photo sont montés sur trépied, la chambre photographique (de format $13 \times 18 \mathrm{~cm}$ ) est en bois, et il faut transporter aussi les châssis et les plaques de verre recouvertes d'une émulsion de collodion, ce qui est extrêmement encombrant. Or, Oskar est asthmatique et s'essouffle vite à l'effort quand il se déplace avec cet équipement. C'est alors qu'en 1913, il a l'idée d'utiliser du film de cinéma $35 \mathrm{~mm}$, en le plaçant horizontalement dans une boîte, avec un objectif (un Kino-Tessar de Zeiss - comme pour la caméra cinématographique qu'utilise Mechau), et un obturateur. II développe les images et constate qu'elles sont d'excellente qualité : le premier Leica (contraction de Leitz camera) est né [2].

Les Leitz, père et fils, sont enthousiasmés par ce petit appareil qui ne s'appelle encore que le Ur (Ur signifiant en allemand «primitif »). Oskar l'utilise pour illustrer la chronique de la vie à Wetzlar. En 1914, une demande de brevet est déposée pour cette invention auprès de l'office allemand - le système d'armement couplé à l'avancement du film étant très novateur. Mais elle est refusée au prétexte que deux autres brevets avaient déjà été accordés à Zeiss et au Français Francisque Pascal de Lyon. Seul le brevet sur l'appareil est retenu.

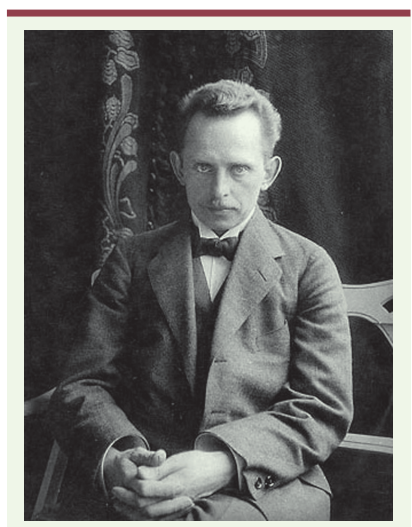

Oskar Barnack (1914). Autoportrait avec le Ur-Leica

La même année, Ernst II emporte ce petit appareil aux États-Unis pour le présenter, mais la guerre éclate et le contraint à rentrer d'urgence.

\section{Les suites de la Première Guerre mondiale}

Au sortir de la guerre, pendant laquelle la firme a travaillé au ralenti, la crise économique et monétaire 
entraîne une poussée inflationniste inouïe. Ernst II, qui a pris la direction de l'Institut à la mort de son père et exporte à l'étranger, crée une monnaie parallèle permettant à ses employés d'acheter des vivres qu'il importe du Danemark.

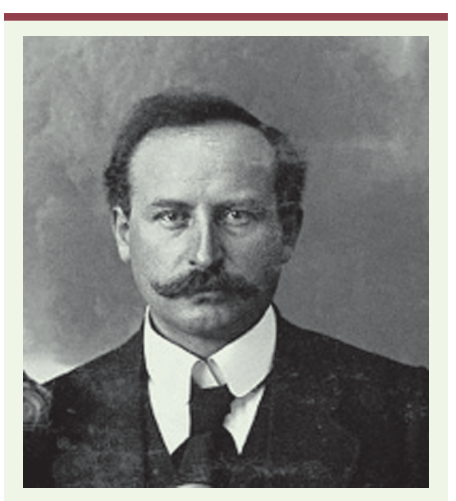

Ernst Leitz II

Sur la colline Kalsmunt, au-dessus de son usine, il a fait construire, en 1917, une grande maison, Haus Friedwart, où il vit avec sa famille et ses trois enfants. L'architecture et surtout les meubles, très jugendstyl $l^{3}$, contrastent avec l'austérité de sa vie et prouvent son ouverture d'esprit sur la modernité de l'Art Nouveau allemand... qui bientôt sera appelé « art dégénéré ».

Pendant ces années d'après guerre, Barnack a continué à perfectionner le Leica. Grâce à Max Berek, un physicien, professeur à Marburg qui travaille aussi pour Leitz, le Leica possède maintenant un objectif, Anastigmat $50 \mathrm{~mm}$, plus net et plus lumineux que les lentilles pré-existantes. En 1925, le Leica 0 est présenté à la foire de Leipzig. Prudemment, Ernst n'en a fait fabriquer qu'une série de 32. Devant le peu d'enthousiasme pour ce petit boîtier de la taille d'un agenda, il se dit qu'il n'avait pas eu tort. Pourtant, certains photojournalistes, comme Eric Salomon, qui rêve justement «de faire des photos chocs pour saisir le naturel », sont séduits. Peu à peu, sa discrétion, sa maniabilité finissent par intéresser professionnels et amateurs. Leitz décide alors de relever le pari industriel et de produire l'appareil en série.

Le modèle Leica 1 sera le premier d'une longue généalogie, mais il possède déjà toutes les qualités : léger $(377 \mathrm{~g})$, discret, capable de tirer 36 poses d'affilée, il devient l'appareil photo favori de tous les grands photographes. Désormais la compagnie Leitz a des succursales à l'étranger. En 1924, elle compte 1000 employés.

\footnotetext{
${ }^{3}$ Conçus par Bruno Paul, architecte, décorateur d'intérieur et aussi excellent
} caricaturiste.

\section{La période nazie}

Ernst est très conscient de la montée du nazisme, et il lui est foncièrement hostile. En 1933, année de l'arrivée d'Hitler au pouvoir, il se présente comme candidat aux élections dans les rangs de l'exparti libéral de gauche, DDP. Pour le régime nazi, la compagnie Leitz, comme la maison Zeiss, est une image de marque. Le ministre de la propagande, Joseph Göbbels, recommande l'utilisation de ces petits appareils aux PK (Propaganda Kompanie). Elles renvoient les images idylliques de la race arienne, fierté du Troisième Reich. Plus tard, c'est aussi avec ces mêmes appareils que la PK 689 immortalisera l'agonie du ghetto de Varsovie.

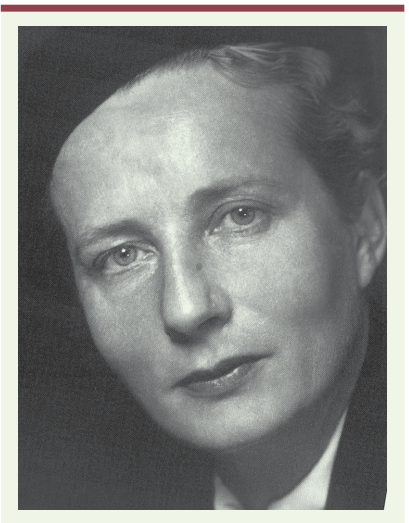

Elsie Kuhn-Leitz

Leitz fabrique aussi du matériel pour l'armée, entre autres l'optique des mécanismes de réglage des fusées V2. Ernst se sent surveillé par la Gestapo et reste le plus discret possible. Les nazis ne sont pas dupes mais préfèrent conserver le directeur pour préserver la bonne marche de l'usine et bénéficier des devises qu'elle rapporte. De fait, Ernst réussit à faire sortir d'Allemagne des Juifs jusqu'en 1939, date à laquelle les frontières sont définitivement fermées. II fait libérer Alfred Türk, son responsable des ventes qui vient d'être arrêté pour avoir tenté d'aider un Juif à fuir. En 1942, par mesure de prudence, il se résout à adhérer au parti national socialiste, tandis que sa fille, Elsie, essaie de son mieux d'améliorer les conditions de vie des centaines d'Ukrainiennes, travailleuses forcées à l'usine. En 1943, c'est Elsie qui est arrêtée à son tour et internée à la prison de la Gestapo de Francfort ; il faudra trois mois et une rançon probablement importante pour qu'il puisse la faire libérer et qu'elle retrouve enfin ses deux jeunes enfants, Cornélia et Knut.

En avril 1945, la première armée américaine arrive à Wetzlar. La ville est en partie détruite par les bombardements mais les usines Leitz sont relativement épargnées. Pour Ernst Leitz II, à 74 ans, c'est la fin du cauchemar, mais le temps de l'insouciance est révolu depuis longtemps.

\section{Épilogue}

Aujourd'hui, la Haus Friedwart n'est plus habitée. Dans l'usine de Solms, à $10 \mathrm{~km}$ de Wetzlar, on peut voir, présentée sous forme d'arbre 
généalogique, toute I'histoire des Leica, du primitif Ur jusqu'aux plus récents modèles, en passant par les reflex et les $M$ (messucher : calcul de la distance) avec visée télémétrique, fabriqués à partir de 1954. Les Leica furent la mémoire $\mathrm{du} x \mathrm{x}^{\mathrm{e}}$ siècle, siècle tragique, avec les reportages de Robert Capa, depuis la guerre d'Espagne jusqu'à l'Indochine, ou siècle familier et rieur avec les gamins et les petites gens des photos prises à la sauvette par Henri Cartier Bresson.

Mais, malgré les millions de boîtiers vendus, la firme Leitz a eu quelques difficultés à évoluer vers le numérique, à s'adapter à la concurrence. Depuis 1987, elle a fusionné avec la firme suisse de microscopes Wild. Sans doute serait-elle tombée dans l'oubli si n'étaient revenus, comme un boomerang, les témoignages de ces jeunes Juifs, recrutés comme apprentis à Wetzlar, puis envoyés à l'étranger avec une lettre de recommandation. La plupart arrivaient à New York à la succursale de Leica dans la Ve avenue. Là, le responsable, Alfred Boch, les accueillait jusqu'à ce qu'on leur trouve un travail. Un jeune employé de 25 ans, Norman Lipton, les a vu arriver, par dizaines, un peu perdus, avec leur Leica autour du cou. Parfois, c'étaient des familles. Mais quand Boch voulut témoigner, après la guerre, la famille Leitz lui demanda d'attendre. Et ce n'est que dans les années 2000 qu'aux États-Unis [3, 4] et en Europe [5-8] la grande presse se fit l'écho de ce qui était resté, dans la famille Leitz, confidentiel : Knut, le fils d'Elsie ne savait presque rien de son grand-père. Le vide est comblé : on dispose aujourd'hui de livres $[9,10]$ et même d'une vidéo [11] qui témoignent de cet héroïsme discret.

On le voit, l'histoire des deux firmes n'a pas suivi des chemins parallèles. Mais les biologistes, et les amoureux du Leica, pour qui les noms de Zeiss et de Leitz sont familiers, seront peut-être heureux de ce petit détour par l'histoire. $\diamond$

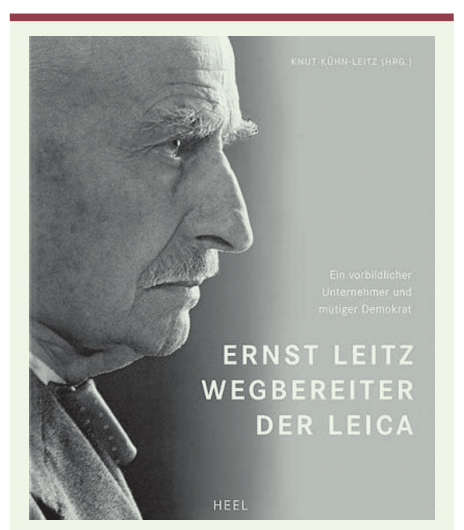

TIRÉS À PART

S. Gilgenkrantz

\section{SUMMARY}

Greatness and tribulations

of two German optic companies.

\section{The Leitz company}

A parallel is drawn between the histories of the two most famous German optic companies. Born in the middle of the $19^{\text {th }}$ century, Zeiss and Leitz went through National Socialism. But their histories are very different. Leitz Archive documents abound, especially from one of the main actors, Oskar Barnack (father of the Leica). Ernst Leitz II and his daughter Elsie tried to help the Jewish workers, their families and, later, the Ukrainian women working in the factory. Leitz succeeded to establish what has become known among historians of the Holocaust as «the Leica Freedom Train ». Their tribulations throw light on the German adventure during the last century. $\diamond$

\section{RÉFÉRENCES}

1. Gilgenkrantz S. Grandeur et vicissitude de deux gandes entreprises d'optique allemandes. I. La firme Zeiss. Med Sci (Paris) 2011 ; 27 : 204-7.

2. Pont PH. Leica saga. Biarritz: Du Pecari, 2002 : 130 p.

3. Smith FD. Ernst Leitz of Wetzlar and Altruism During the Holocaust. http:// nemeng.com/leica/005eb.shtml (2002) 1 .

4. Gilbert G. The Leica freedom train. http://www.youtube.com/ watch?v=hKxGbNXt_Is\&feature=related

5. Honigsbaum M. New life through a lens. Financial Times, 2 février 2007

6. Connolly K. Behind the camera: secret life of man who saved Jews from Nazis. The Guardian, 10 février 2007.

7. Unternehmer Ernst Leitz: Der andere Schindler. Die Welt, 9 février 2007.

8. Langellier JP. Au bout de l'objectif la liberté. Le Monde, 17 février 2007.

9. Kühn-Leitz K. Ernst Leitz. Ein Unternehmer mit Zivilcourage während der Zeit des Nationalsozialismus. Hanau : Cocon-Verlag, 2008 : 128 p.

10. Porezag K. Ernst Leitz aus Wetzlar und die Juden. Mythos und Fakten. Berlin : Metropol, $2009: 240 p$

11. http://www.youtube.com/watch?v=hKxGbNXt_Is

\section{CONFLIT D'INTÉRÊTS}

L'auteur déclare n'avoir aucun conflit d'intérêts concernant les données publiées dans cet article.

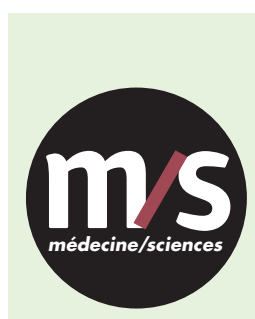

Tarifs d'abonnement $m / s-2010$

$>$ Grâce à $m / s$, vivez en direct les progrès des sciences biologiques et médicales

Abonnez-vous

à médecine/sciences
Bulletin d'abonnement page 368 dans ce numéro de $\mathrm{m} / \mathrm{s}$

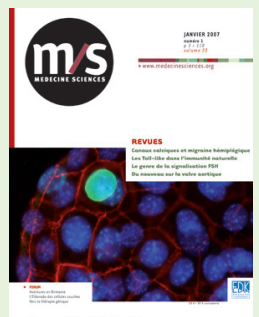

